\title{
1 Uhrf1 governs the proliferation and differentiation of muscle satellite cells
}

3 Hiroshi Sakai ${ }^{1,2,10^{*}}$, Yuichiro Sawada ${ }^{3,10}$, Naohito Tokunaga $^{4}$, So Nakagawa ${ }^{5}$, Iori Sakakibara ${ }^{6}$,

4 Yusuke Ono $^{7}$, So-ichiro Fukada ${ }^{8}$, Tadahiko Kikugawa ${ }^{3}$, Takashi Saika ${ }^{3}$, Yuuki Imai ${ }^{1,2,9,11^{*}}$

$6{ }^{1}$ Division of Integrative Pathophysiology, Proteo-Science Center, Ehime University, Toon, Japan.

$7 \quad{ }^{2}$ Department of Pathophysiology, Ehime University Graduate School of Medicine, Toon, Japan.

$8 \quad{ }^{3}$ Department of Urology, Ehime University Graduate School of Medicine, Toon, Japan.

$9 \quad{ }^{4}$ Division of Analytical Bio-Medicine, Advanced Research Support Center, Ehime University,

10 Toon, Japan.

$11{ }^{5}$ Department of Molecular Life Science, Tokai University School of Medicine, Kanagawa, Japan

$12{ }^{6}$ Department of Nutritional Physiology, Institute of Medical Nutrition, Tokushima University

13 Graduate School, Tokushima, Japan.

$14{ }^{7}$ Department of Muscle Development and Regeneration, Institute of Molecular Embryology and

15 Genetics, Kumamoto University, Kumamoto, Japan.

$16{ }^{8}$ Project for Muscle Stem Cell Biology, Graduate School of Pharmaceutical Sciences, Osaka

17 University, Osaka, Japan.

$18{ }^{9}$ Division of Laboratory Animal Research, Advanced Research Support Center, Ehime University,

19 Toon, Japan.

$20{ }^{10} \mathrm{Co}$-first author

$21 \quad{ }^{11}$ Lead contact

$22{ }^{*}$ Correspondence: sakai.hiroshi.wh@ehime-u.ac.jp (H.S.), y-imai@m.ehime-u.ac.jp (Y.I.) 


\section{Summary}

2 DNA methylation is an essential form of epigenetic regulation responsible for cellular identity. In

3 muscle stem cells, termed satellite cells, DNA methylation patterns are tightly regulated during

4 differentiation. However, it is unclear how these DNA methylation patterns are maintained. We

5 demonstrate that a key epigenetic regulator, ubiquitin like with PHD and RING finger domains 1

6 (Uhrf1), is activated in proliferating myogenic cells but not expressed in quiescent or differentiated

7 myogenic cells in mice. Ablation of Uhrf1 in mouse satellite cells impairs their proliferation and

8 differentiation, leading to failed muscle regeneration. Loss of Uhrf1 in satellite cells alters

9 transcriptional programs, leading to DNA hypomethylation with activation of Cdkn1a and Notch

10 signaling. Although down-regulation of Cdknla rescued proliferation but not differentiation,

11 inhibition of Notch signaling rescued impaired differentiation of Uhrf1-deficient satellite cells.

12 These findings point to Uhrfl as a regulator of self-renewal and differentiation of satellite cells via

13 genome-wide DNA methylation patterning.

\section{Keywords}

16 Skeletal muscle stem cells, satellite cells, UHRF1, DNA methylation, muscle regeneration 


\section{Introduction}

Adult skeletal muscle has a great capacity for regeneration, which is mediated by muscle satellite (stem) cells that express the Pax7 gene (Seale et al., 2000). Genetic ablation of Pax7-expressing satellite cells in mice led to a drastic loss of muscle tissue and loss of muscle regeneration (Lepper et al., 2011; McCarthy et al., 2011; Murphy et al., 2011; Sambasivan et al., 2011). During regeneration, satellite cells are rapidly activated to proliferate and fuse to form new skeletal muscle fibers. Although it has been reported that many intrinsic and extrinsic cell signals contribute to the maintenance, activation, and differentiation of satellite cells, little information is available on the role of epigenetic regulation, especially maintenance of DNA methylation patterns, in satellite cells during muscle regeneration.

DNA methylation is critical for regulating gene transcription. Several studies reported that DNA methylation patterns are altered during the proliferation and differentiation of mouse and human myogenic cells (Carrió et al., 2015; Miyata et al., 2015; Tsumagari et al., 2013). A family of DNA methyltransferases (DNMT) mediate DNA methylation by enzymatic activities including de novo methylation (catalyzed by DNMT3a and DNMT3b) or maintenance of methylation (by DNMT1). Deleting DNMT3a in satellite cells led to loss of proliferation with increased expression of the Cdknlc gene (Naito et al., 2016). Further, we reported that deletion of Dnmt1 in satellite cells impaired muscle regeneration in vivo, resulting in a reduced number of satellite cells in vitro (Iio et al., 2021). However, it remains largely unknown how the DNA methylation pattern of satellite cells is maintained during proliferation and differentiation.

23 methylation by recruiting DNMT1 to hemi-methylated DNA sites (Bostick et al., 2007; Sharif et al., 24 2007), and it also can interact with DNMT3a and DNMT3b (Meilinger et al., 2009). Consistently, UHRF1 is up-regulated in proliferating cells, such as neural stem and basal stem cells in the airway, 
1 and down-regulated during differentiation or quiescence (Ramesh et al., 2016; Xiang et al., 2017).

2 Previous reports have shown that ablation of $U h r f 1$ in different cell types, including embryonic

3 stem cells (Sharif et al., 2007), T cells (Obata et al., 2014), hematopoietic stem cells (Zhao et al.,

4 2017), chondrocytes (Yamashita et al., 2017), and adult neural stem cells (Blanchart et al., 2018),

5 leads to DNA hypomethylation, resulting in abrogation of proliferation and/or differentiation.

6 However, no information is available on the functions of Uhrfl in satellite cells in muscle

7 regeneration.

9 regeneration. Deletion of $U h r f l$ in satellite cells led to impaired muscle regeneration with loss of

10 satellite cells in mice. Uhrfl-deficient satellite cells exhibited dramatic changes in transcript levels

11 and genome-wide DNA methylation profiles, which led to up-regulation of Cdkn1a and genes

12 involved in Notch signaling. Therefore, we show that Uhrfl is critical for controlling the

13 proliferation and differentiation of satellite cells via DNA methylation.

\section{Results}

16 Uhrf1 is active in satellite cells after muscle injury and during in vitro culture.

17 To determine whether and when Uhrfl is expressed in muscles, the tibialis anterior (TA) muscles 18 were injured by cardiotoxin (CTX) injection; uninjured and injured TA muscles were collected on 19 different days post injury (dpi). During muscle regeneration, the Uhrfl transcript level peaked at 4 20 dpi and then decreased by 14 dpi (Figure S1A). To determine which myogenic cells contain active 21 Uhrf1, immunofluorescence staining of Uhrf1 and Pax7 was performed in TA muscle sections

22 (Figure 1A). In uninjured TA muscles, almost none of the Pax7+ satellite cells expressed Uhrf1

23 (Figure 1B). At 5 dpi, when the number of Pax 7+ cells peaked during normal regeneration post-

24 injury (Murphy et al., 2011), 11\% of Pax7+ cells expressed Uhrf1, and this percentage decreased to $255.8 \%$ at 14 dpi (Figure 1B), which was compatible with the Uhrf1 transcript level in injured TA 
muscles. In addition, Uhrfl expression gradually decreased after induction of differentiation in C2C12 cells (Figures S1B-D).

To further examine the expression pattern of Uhrf1, satellite cells were isolated by FACS

4 and cultured for up to 7 days in vitro (Figure 1C). Almost all cells were positive for Pax7 on day 1,

5 but none of these cells expressed Uhrfl (Figure 1D), which supported the in vivo results in intact

6 muscles. The majority of myogenic cells (78\%) were positive for Uhrf1 on day 3, and this

7 percentage gradually decreased during differentiation (Figure 1E). Whereas $87 \%$ of the Uhrf1+

8 cells expressed Ki67 on day 3 (Figure 1F), only 6.9\% of Myogenin+ cells (differentiated) expressed

9 Uhrf1 on day 4 (Figure 1G), and almost no Myh1+ myotubes expressed Uhrf1 (Figure 1H). These

10 observations indicate that Uhrf1 is not expressed in satellite cells during quiescence but is

11 transiently expressed in proliferating myogenic cells before being down-regulated during

12 differentiation.

\section{Loss of Uhrf1 in satellite cells impairs muscle regeneration.}

To determine whether Uhrfl expression in satellite cells is necessary for muscle regeneration, we selectively deleted this gene using $\operatorname{Pax}^{\mathrm{CE} /+}$ (Lepper et al., 2009) and Uhrfl ${ }^{f l f l}$ (Skarnes et al., 2011) mice. The efficiency of Uhrfl ablation in satellite cells in $\operatorname{Pax} 7^{C E /+} ; U h r f 1^{f l f l}$ mice was measured by isolating satellite cells by FACS from hindlimb muscles of $\mathrm{Pax}^{\mathrm{CE/+}}$;Uhrfl $\mathrm{I}^{\mathrm{fl} f \mathrm{l}}$ mice following administration of oil (control mice) or tamoxifen (TMX, mutant mice) for 5 consecutive days (Figures S2A and S2B). Deletion was highly efficient as the Uhrf1 transcript level was 90\% lower

21 in mutant mice than control mice (Figure S2C). Uhrf1 protein was expressed in $65 \%$ of satellite cells cultured for 3 days from control mice but in 5.2\% of those from mutant mice (Figures S2D and

23 S2E). Together, these experiments indicate that Uhrf1 expression was effectively down-regulated in 24 satellite cells from $\operatorname{Pax}^{C E /+} ; U h r f I^{f l f l}$ mice. 
To investigate the effect of Uhrf1 expression loss in satellite cells on muscle regeneration, the TA muscles of control and mutant mice were injured by CTX and harvested at 5 dpi (Figure 2A). As $P a x 7^{C E /+}$ mice treated with TMX (Cre control mice) exhibit a mild muscle regeneration

4 phenotype (Mademtzoglou et al., 2018; Sakai et al., 2020), Cre control mice were included in the

5 experiments for regeneration. The regenerating fibers, characterized by expression of embryonic myosin heavy chain (Myh) 3, were detected at 5 dpi in control mice (Figure 2B), similar to a previous report (Murphy et al., 2011). Although there was no difference in the mass (Figure S2F) or cross-sectional area (CSA) of TA muscles (Figure S2G), the Myh3 level was decreased in Cre control mice compared with the control, while the proportion of Myh3+ regenerating fibers was severely reduced in mutant mice (Figure 2C). These results indicate that loss of Uhrfl affects the regeneration of new fibers. In addition, there was a significant decrease in the number (Figure 2E) and proliferation of Pax7+ cells (Figure 2F) at 5 dpi in mutant mice compared with control and Cre control mice.

To determine whether the observed phenotype at 5 dpi was sustained during the muscle regenerative process, injured TA muscles were harvested at 28 dpi (Figure 2A), at which time regeneration is largely complete (Murphy et al., 2011). Although there was no difference in the TA muscle mass between control and Cre control muscle according to macroscopic observations (Figure 2G), the mutant mice exhibited significant muscle mass loss in the TA muscles (Figure 2H).

19 There was a significant decrease in the total CSA in mutant mice (Figures 2I and S2H) but no 20 difference between the control and Cre control mice. Also, TA sections contained a significantly

21 lower number of Pax $7+$ cells in mutant mice compared with control mice (Figure $2 \mathrm{~K}$ ), and this 22 significant decrease remained even after normalization to the total CSA (Figure 2L). In summary, 23 loss of Uhrf1 expression in satellite cells significantly impaired proliferation and muscle 24 regeneration. 
To investigate the mechanisms of satellite cell impairment in $P a x 7^{C E /+} ; U h r f 1^{f l f l}$ mice, single myofibers were isolated from the extensor digitorum longus muscle of control and mutant mice and cultured for up to 4 days (Figure S2I). On days 0 and 2, the number of Pax7+ satellite cells per myofiber was comparable between the mutant and control mice (Figure S2J). In contrast, the total number of satellite cells and the proportion of Pax7-MyoD+ cells, which are committed to activation and differentiation, were decreased in the myofibers of mutant mice compared with control mice (Figure S2J).

To further characterize the impaired proliferation and differentiation observed in culture,

9 satellite cells from control and mutant mice were characterized in vitro (Figure S2K). In EdU

10 labelling experiments to assess proliferation, approximately $70 \%$ of satellite cells were positive for

11 EdU in control mice, compared with $33 \%$ in mutant mice, on day 3 (Figures S2L and S2M).

12 Furthermore, the number of Myogenin+ cells was significantly decreased in mutant mice on day 4

13 (Figures S2N and S2O). In addition, myotube formation, observed by Myh1 staining on day 7, was

14 also compromised in the mutant mouse cells (Figures S2P and S2Q). Taken together, these in vitro

15 findings indicate that Uhrf1 is required in myogenic cells for proliferation but not activation, and

16 the loss of Uhrfl leads to impairments of differentiation.

\section{Loss of Uhrf1 in satellite cells alters DNA methylation patterns and subsequently}

transcriptional programs.

21 obtained from control and mutant mice (Figure 3A). A heatmap of the expression profiles showed a clear distinction between the control and mutant satellite cells (Figure S3A). We detected 2044 upregulated and 1833 down-regulated genes in the mutant compared with control satellite cells (Figures 3B and S3B). According to Gene Ontology (GO) enrichment analysis, the down-regulated 
1 (Figure 3C), consistent with the impairment of these processes observed in the mutant cells. The genes that were up-regulated in mutant satellite cells showed enrichment of GO terms associated with "vasculature development". In addition, several gene sets associated with impaired cell proliferation or differentiation, such as "positive regulation of proteolysis" and "apoptotic signaling pathway", were also enriched among the up-regulated genes (Figure 3C). However, only caspase 9 in the critical apoptotic signaling pathway was up-regulated in the mutant cells (Table 1). These results suggest that activation of apoptosis may not be a main cause of the phenotype of the mutant satellite cells.

To determine whether the genes up-regulated by Uhrf1 deficiency were specific to satellite cells, we compared the up-regulated genes in chondrocytes (Yamashita et al., 2017) and

11 hematopoietic stem cells lacking Uhrfl (Zhao et al., 2017) with those in satellite cells (Figure S3C). Among the 2044 genes up-regulated in Uhrf1 deficient-satellite cells, 331 overlapped with the 1122 genes up-regulated in Uhrfl-knockout chondrocytes. On the other hand, only 77 genes overlapped with the 626 genes up-regulated in hematopoietic stem cells lacking Uhrfl. These results indicate that Uhrfl deficiency induces up-regulation of partially overlapped genes, but cell-specific Most DNA methylation occurs at transposable elements (TEs) within the mouse genome. knockout of Uhrf1 may lead to up-regulation of cell-specific genes. of TEs using RNA-Seq data using STAR program (v2.7.6a) (Dobin et al., 2013) with dedicated Loss of DNA methylation due to Uhrfl deficiency in embryonic stem cells (Sharif et al., 2007) or parameters and algorithms for TE analysis (see Materials and Methods). As expected, several TEs

23 We found up-regulation of intracisternal A particles (IAPs) in mutant compared with control 24 satellite cells (Figure S3E), which is in line with the results from Uhrfl-deficient embryonic stem 25 cells (Bostick et al., 2007; Sharif et al., 2007). 
We next performed methyl-CpG binding domain protein 2-enriched genome sequencing (MBD2-Seq) in the control and mutant satellite cells (Figure 3A). The higher GC count per read in methylated DNA compared with unmethylated DNA (Figure S3F) and the distinct methylation

4 patterns observed at the $\mathrm{H} 19$ and Kcnqlot 1 loci, which are imprinted genes (Figure S3G), indicated 5 proper enrichment of methylated DNA by MBD2. To identify DNA loci methylated by Uhrf1, peak calling was performed using MACS2. We identified 39,231 peaks, and these loci were enriched in promoters, downstream regions, and gene bodies of the genome (Figure 3D). This change in the DNA methylation profile might not be a secondary result of decreased Dnmt expression, as the

9 expression of Dnmt1, Dnmt $3 a$, and Dnmt $3 b$ did not change drastically compared with Uhrf1 10 deficiency in mutant satellite cells (Figure S3H), as determined by RNA-Seq. To identify Uhrf1 target genes in satellite cells, we analyzed the genes up-regulated by Uhrfl deficiency that overlapped with the Uhrf1-mediated DNA methylated peaks. We found 222 genes with Uhrf1mediated peaks within $-5000 \mathrm{bp}$ from the transcriptional start site (Figure 3E). GO enrichment analysis showed that these genes are associated with "vasculature development" and "cell cycle 16 (Figure 3F). Among these genes, Cdknla was a candidate mediator of "cell cycle arrest" (Table 1), consistent with the reduced proliferation of the mutant satellite cells. We also found that genes annotated as "vasculature development" included those related to Notch signaling that play critical roles in sustaining the quiescent state of satellite cells (Fukada et al., 2011), and their expression was up-regulated in the mutant satellite cells (Figure 3G). We also detected reduced DNA methylation levels in the regions upstream of the Cdknla and Notch3 loci in mutant satellite cells (Figure 3H). These results suggest that $U h r f 1$ deficiency alters DNA methylation patterns, followed by transcriptional changes in satellite cells. 
1 Suppression of Cdkn1a and Notch signaling rescued the impaired proliferation and differentiation of Uhrf1-deficient satellite cells.

3 To explore whether up-regulation of the Cdkn1a or Notch signaling pathway is responsible for the

4 impaired proliferation and differentiation of Uhrfl-deficient satellite cells, we treated mutant

5 satellite cells with siRNA targeting $C d k n l a$ or with DAPT, an inhibitor of $\gamma$-secretase (Figure 4A).

6 Although Cre control satellite cells showed up-regulation of Cdkn la (Figure S4A) and reduced

7 proliferation compared with control mice, siRNA knockdown of Cdknla in mutant satellite cells

8 (Figure S4A) significantly rescued the impaired proliferation of the mutant satellite cells (Figures

9 4B and 4C). However, Cdknla knockdown was not sufficient to rescue the impaired differentiation

10 of the mutant satellite cells (Figures 4D and 4E). In contrast, although DAPT treatment did not

11 affect the proportion of EdU+ cells (Figures 4F and 4G), unlike siRNA-mediated knockdown of

12 Cdknla, it partially rescued proliferation in terms of absolute cell numbers (Figure S4C), and

13 DAPT treatment suppressed HeyL expression (Figure S4B) and rescued differentiation, as

14 measured by Myogenin expression (Figures 4H and 4I), in mutant satellite cells. We also confirmed 15 that knockdown of Cdknla (Figures S4D and S4E) and DAPT treatment (Figures S4F and S4G) did 16 not selectively expand the population of surviving Uhrf1+ cells among mutant satellite cells. Taken 17 together, Uhrf1-mediated DNA methylation negatively regulated the expression of Cdkn1a and 18 Notch signaling for physiological proliferation and/or differentiation of satellite cells.

\section{Discussion}

21 In this study, we identified Uhrfl as a critical regulator of skeletal muscle satellite cells. Notably, Uhrfl is not expressed during quiescence but is up-regulated during proliferation and downregulated during differentiation in satellite cells. Our findings agree with previous reports showing

24 that Uhrf1 is necessary for maintaining the DNA methylation profile during DNA replication in 
up-regulated during proliferation and down-regulated during differentiation or quiescence (Ramesh et al., 2016; Xiang et al., 2017). As Uhrfl overexpression was reported to contribute to cancer initiation and progression (Mudbhary et al., 2014), tight regulation of Uhrfl expression in stem cells appears to be critical for self-renewal and differentiation.

Further, ablation of Uhrf1 in satellite cells led to impaired muscle regeneration, similar to Dnmt1 - (Iio et al., 2021) or Dnmt3a-deficient mice (Naito et al., 2016). The marked loss of EdU incorporation indicated a remarkable reduction in the number of Uhrfl-deficient satellite cells in vivo and in vitro at $28 \mathrm{dpi}$. Therefore, the impairment of muscle regeneration of mutant mice could result from decreased numbers of myogenic cells.

In addition, we report that loss of Uhrfl in satellite cells alters transcriptional programs caused by alteration of genome-wide DNA methylation patterns. Consistent with the impaired proliferation of $U h r f 1$-deficient satellite cells, we found that many cell-cycle-related genes were down-regulated. We propose that Uhrfl deficiency in mutant satellite cells results directly in hypomethylation and general up-regulation of transcription. Indeed, the GO term most enriched among the up-regulated genes by hypomethylation in Uhrfl-deficient satellite cells was "vascular development". As satellite cells have a potential to adopt a pericyte phenotype following stimulation of the Notch and PDGF signaling pathways (Gerli et al., 2019), loss of Uhrf1 in satellite cells might trigger partial transdifferentiation into vascular-like cells. We found that TEs, especially IAPs, were strongly up-regulated in Uhrfl-deficient satellite cells. This phenotype is comparable with that of Uhrfl-deficient cortical neural stem cells showing strong activation of IAPs (Ramesh et

21 al., 2016). Since long-terminal repeats in IAPs could change gene regulation at a given locus

22 (Morgan et al., 1999), further studies are needed to clarify whether transposition of IAPs into new 23 genomic loci would contribute to the phenotype of mutant satellite cells. Finally, we showed that suppression of the increased Cdkn1a and Notch signaling in Uhrfldeficienct satellite cells rescued their impaired proliferation and differentiation. Cdkn1a was 
1 identified as a target of Uhrf1 in neural stem cells and colonic regulatory T cells (Blanchart et al.,

2 2018; Obata et al., 2014). Thus, loss of Uhrfl might lead to cell cycle arrest in diverse cell types.

3 We found that silencing of Cdknla in mutant satellite cells rescued their decreased cell

4 proliferation, but not differentiation, suggesting that $U h r f 1$-mediated satellite cells require

5 additional factors for differentiation. Notch signaling plays a critical role in sustaining the quiescent

6 state of satellite cells. Notch3 is highly expressed in quiescent satellite cells (Fukada et al., 2007),

7 and its expression is strongly up-regulated by overexpression of the Notch intracellular domain

8 (Wen et al., 2012). These results suggest that cell autonomous Notch signaling inhibits muscle

9 differentiation of mutant satellite cells. Thus, we propose that Uhrf1 epigenetically regulates, at

10 least in part, satellite cell proliferation via Cdkn1a-mediated cell cycle regulation, and loss of Uhrfl

11 in myogenic cells leads to up-regulation of Notch signaling and thereby decreased differentiation

12 and proliferation of satellite cells.

13 In conclusion, the present study demonstrates that Uhrf1 is a critical epigenetic regulator of

14 proliferation and differentiation in myogenic cells, by controlling gene expression via maintenance

15 of DNA methylation.

\section{Acknowledgments}

18 We thank S. Tajbakhsh for critically reading the manuscript, S. Nakanishi and A. Nishio for their

19 technical support, K. Kameda for the flow cytometry support and other members of the Division of 20 Analytical Bio-Medicine and the Division of Laboratory Animal Research, the Advanced Research

21 Support Center, Ehime University. This work was supported in part by MEXT/JSPS KAKENHI (JP18H06439 and JP19K19947 to H.S.; JP23689066, JP15H04961, JP15K15552, JP17K19728,

23 JP19H03786 to Y.I.); HIRAKU-Global Program, which is funded by MEXT's “Strategic

24 Professional Development Program for Young Researchers" (to H.S.); and The Nakatomi

25 Foundation and Takeda Science Foundation (to H.S. and Y.I.). 


\section{Author Contributions}

3 Conceptualization, H.S. and Y.I. Methodology, H.S., S.N., I.S., Y.O., and S.F. Software, H.S., N.T.,

4 and S.N. Validation, H.S. and Y.S. Formal analysis, H.S., N.T., and S.N. Investigation, H.S., Y.S.,

5 and N.T. Resources, Y.O. and S.F. Data curation, H.S. and N.T. Writing - original draft, H.S. and

6 Y.I. Writing - review \& editing, H.S. and Y.I. Visualization, H.S. and Y.S. Supervision, H.S., T.K.,

$7 \quad$ T.S., and Y.I. Project administration, Y.I. Funding acquisition, H.S., T.K., T.S., and Y.I.

8

\section{Declaration of Interests}

10 The authors declare no competing interests. 


\section{Figure Legends}

Figure 1. Uhrf1 is active in satellite cells after muscle injury and during in vitro culture. (A)

Immunostaining of Pax 7 and Uhrf1 during muscle regeneration. Arrows show Uhrf1+Pax7+ cells.

(B) Quantification of Uhrf1+ cells among Pax7+ cells in uninjured and injured TA muscles

collected at 5 and 14 dpi. (C) Time-course of satellite cells cultured in vitro. (D) Immunostaining of Uhrf1, Pax7, Ki67, Myogenin, and Myh1 after 1, 3, 4, and 7 days of culture. (E) Quantification of Uhrf1+ cells in vitro. (F) Quantification of Uhrf1+ cells among proliferating (Ki67+) cells after 3 days of culture. (G) Quantification of Uhrf1+ cells among Myogenin+ cells after 4 days of culture. (H) Quantification of Uhrf1+ cells in Myh1+ fibers after 7 days of culture. For all graphs, data were pooled from three independent experiments and are expressed as the mean with $95 \%$ CI. Scale bars

$11=20 \mu \mathrm{m}$. See also Figure $\mathrm{S} 1$.

Figure 2. Loss of Uhrf1 expression in satellite cells impairs muscle regeneration. (A)

Experimental design for TMX treatment followed by CTX injection and sample harvest at 5 and 28 dpi. (B) Immunostaining of Myh3 in TA muscles at 5 dpi. (C) The average area of Myh3+ regenerating myofibers in TA muscle cross-sections at 5 dpi ( $n=3$ or 5 mice/condition). (D) Immunostaining of Pax7 in TA muscle cross-sections at 5 dpi. (E) Quantification of Pax 7+ cells at 5 dpi ( $\mathrm{n}=6$ mice/condition). (F) Quantification of Pax7+EdU+ cells at $5 \mathrm{dpi}(\mathrm{n}=6$ mice/condition). (G) Whole mount TA at 28 dpi. (H) Mass of TA muscles at 28 dpi ( $\mathrm{n}=4$ or 5 mice/condition). (I) The average total area of TA muscle cross-sections at 28 dpi $(n=3$ or 4 mice/condition). (J)

21 Immunostaining of Pax7 in TA muscle cross-sections at 28 dpi. (K, L) The total and average number of Pax7+ cells in TA muscle cross-sections at $28 \mathrm{dpi}\left(\mathrm{n}=4\right.$ mice/condition). ${ }^{*} p<0.05,{ }^{* *} p$ $<0.01, * * * p<0.001, * * * * p<0.0001$. Scale bars: $50 \mu \mathrm{m}$ in (D and J), $500 \mu \mathrm{m}$ in (B), and $50 \mathrm{~mm}$ in (G). See also Figure S2. 
1 Figure 3. Loss of Uhrf1 in satellite cells alters DNA methylation patterns and thereby transcriptional programs. (A) Experimental design for RNA-Seq and MBD2-Seq. (B) Heatmap of the expression values (normalized as transcripts per million, TPMs) of the top 100 differentially expressed genes in each sample. (C) Bar graph of the Gene Ontology (GO) terms enriched among the up-regulated and down-regulated genes in mutant satellite cells. (D) Distribution of Uhrf1mediated methylated DNA with the given intervals and scores, with the genomic features determined using a cis-regulatory element annotation system. (E) Venn diagram of the up-regulated genes associated with Uhrfl-mediated methylated DNA loci. (F) Bar graph of the GO terms enriched among the up-regulated genes associated with Uhrfl-mediated methylated DNA loci. (G) RNA-Seq analyses of Notch and myogenic-related genes (normalized as TPMs) in each sample.

11 Genes with an asterisk were not differentially expressed. $(\mathrm{H})$ Methylated DNA signals around the Cdknla and Notch3 loci in control and mutant satellite cells visualized using IGV. See also Figure S3.

\section{Figure 4. Decreased Cdkn1a and Notch signaling rescues the impaired proliferation and} differentiation of Uhrf1-deficienct satellite cells. (A) Experimental design for the siRNA and DAPT treatments. (B) EdU detection in Cdknla-knockdown satellite cells. (C) Quantification of EdU $+C d k n 1 a$-knockdown satellite cells ( $\mathrm{n}=5$ for control and Cre control; $\mathrm{n}=6$ for mutant cells). (D) Immunostaining of Myogenin in Cdknla-knockdown satellite cells. (E) Quantification of Myogenin $+C d k n l a$-knockdown satellite cells $(\mathrm{n}=5$ for control; $\mathrm{n}=3$ for Cre control; $\mathrm{n}=6$ for mutant cells). (F) EdU detection in satellite cells treated with DAPT. (G) Quantification of EdU+

23 Immunostaining of Myogenin in satellite cells treated with DAPT. (I) Quantification of Myogenin+ DAPT-treated satellite cells ( $\mathrm{n}=4$ for control; $\mathrm{n}=3$ for Cre control; $\mathrm{n}=6$ for mutant cells). ${ }^{*} p<$ $0.05, * * p<0.01, * * * p<0.001$. Scale bars $=50 \mu \mathrm{m}$. See also Figure S4. 


\section{Tables}

2 Table 1. Analysis of senescence- and apoptosis-related gene expression by RaNA-Seq.

\begin{tabular}{|l|l|l|l|l|l|l|}
\hline Symbol & ExpMea & Exp & Exp Mutant & log2FC & p-value & padj \\
\hline Cdkn1a & 600.15 & 473.807 & 726.494 & 0.616 & $4.8 \mathrm{e}-5$ & $6.0 \mathrm{e}-4$ \\
\hline Cdkn1b & 25.838 & 27.355 & 24.321 & -0.163 & 0.064 & 0.242 \\
\hline Cdkn2b & 9.607 & 8.206 & 11.007 & 0.383 & 0.079 & 0.283 \\
\hline Cdkn2a & 18.511 & 17.514 & 19.509 & 0.148 & 0.487 & 0.85 \\
\hline Trp53 & 165.366 & 163.689 & 167.043 & 0.029 & 0.8 & 0.926 \\
\hline Casp9 & 5.16 & 4.78 & 5.54 & 0.178 & 0.004 & 0.029 \\
\hline Casp6 & 13.381 & 12.815 & 13.946 & 0.114 & 0.024 & 0.114 \\
\hline Casp8 & 18.767 & 17.74 & 19.794 & 0.15 & 0.217 & 0.555 \\
\hline Casp7 & 7.432 & 7.88 & 6.984 & -0.154 & 0.288 & 0.656 \\
\hline Casp3 & 36.873 & 36.342 & 37.404 & 0.04 & 0.599 & 0.917 \\
\hline
\end{tabular}


1 Table 2. Oligonucleotides used in this study.

\begin{tabular}{|l|l|l|}
\hline Oligo name & Sequence & Additional information \\
\hline Uhrf1_F & CCACACCGTGAACTCTCTGTC & qPCR \\
\hline Uhrf1_R & GGCGCACATCATAATCGAAGA & qPCR \\
\hline MyoD1_F & CACTACAGTGGCGACTCAGATGCA & qPCR \\
\hline MyoD1_R & CCTGGACTCGCGCGCCGCCTCACT & qPCR \\
\hline Myog_F & CAACCAGGAGGAGCGCGATCTCCG & qPCR \\
\hline Myog_R & GGCGCTGTGGGAGTTGCATTCACT & qPCR \\
\hline Cdkn1a_F & CCTGGTGATGTCCGACCTG & qPCR \\
\hline Cdkn1a_R & CCATGAGCGCATCGCAATC & qPCR \\
\hline HeyL_F & GTCTTGCAGATGACCGTGGA & qPCR \\
\hline HeyL_R & CTCGGGCATCAAAGAACCCT & qPCR \\
\hline
\end{tabular}


Methods

\section{Animals}

3 C57BL/6JJcl mice were purchased from CLEA Japan. The Pax $7^{C E /+}$ (Lepper et al., 2009; Jackson

4 Laboratories, stock no: 012476) and Uhrflflfll $\left(B 6 D n k ; B 6 N-U h r f 1^{<t m 1 a(E U C O M M) W t s i>/} I e g\right.$; Skarnes et

5 al., 2011; European Mouse Mutant Archive) mouse strains have been described previously. Uhrf flffl

6 mice were crossed with $\operatorname{Pax} 7^{C E /+}$ mice to generate $\operatorname{Pax} 7^{C E /+} ; U h r f 1^{f l / f l}$ mice. All mice were housed in

7 a specific pathogen-free facility under climate-controlled conditions and a 12-h light/dark cycle and

8 were provided water and a standard diet ad libitum. All male animals were evaluated at age 7-12

9 weeks old. Tamoxifen $(150 \mu 1,20 \mathrm{mg} / \mathrm{ml})$ (Sigma, cat\# T5648) dissolved in corn oil (Sigma, cat\#

10 C8267) was injected intraperitoneally for 5 consecutive days to induce Cre-mediated

11 recombination.

\section{Muscle regeneration}

14 Injury was induced by injecting $100 \mu 110 \mu \mathrm{M}$ cardiotoxin (CTX) solution (Latoxan, cat\# L8102)

15 into the left tibialis anterior (TA) muscles under anesthesia using isoflurane. The right TA served as

16 the intact control.

\section{Immunofluorescence and microscopy}

19 For immunofluorescence (IF) staining, muscles were frozen in liquid nitrogen-chilled isopentane,

20 and $10 \mu \mathrm{m}$ cryosections were prepared. For Pax7 and Uhrf1 staining, sections were fixed in 4\%

21 paraformaldehyde (PFA) in PBS for $5 \mathrm{~min}$ and then boiled in antigen retrieval buffer (10 $\mathrm{mM}$

22 sodium citrate, $\mathrm{pH}$ 6) for $10 \mathrm{~min}$. For Myh3 staining, tissue sections were fixed for $10 \mathrm{~min}$ in

23 acetone at $-20^{\circ} \mathrm{C}$ before boiling, blocked in 5\% goat serum (Gibco, cat\# 16210-064) in PBS for 60

24 min, and incubated with the primary antibodies overnight at $4{ }^{\circ} \mathrm{C}$. The antibodies used for IF

25 staining were anti-Pax7 (DSHB, cat\# PAX7, RRID:AB_528428; 1/5 dilution; or Invitrogen, cat\# 
PA1-117, RRID:AB_2539886; 1/100 dilution), anti-Uhrf1 (Santa Cruz Biotechnology, cat\# sc373750, RRID: AB_10947236; 1/50 dilution), anti-Myh3 (DSHB, cat\# F1.652, RRID:AB_528358; 1/20 dilution), Alexa fluor 568 goat anti-mouse IgG1 (Invitrogen, cat\# A-21124; 1/1000 dilution), and Alexa fluor 488 goat anti-rabbit IgG (Invitrogen, cat\# A-11008; 1/1000 dilution). permeabilization using $0.5 \%$ Triton X-100 in PBS for 10 min. Cells were blocked using 5\% goat serum in PBS. The following primary antibodies were used: anti-Uhrf1 (Santa Cruz Biotechnology, cat\# sc-373750, RRID: AB_10947236; 1/100 dilution; or MBL, cat\# D289-3 RRID:AB_10597260; 1/100 dilution), anti-Ki67 (Novus, cat\# NB500-170, RRID:AB_10001977; 1/100 dilution), antiMyogenin (Santa Cruz Biotechnology, cat\# sc-12732, RRID:AB_627980; 1/100 dilution), and antiMyh1 (DSHB, cat\# MF20, RRID:AB_2147781; 1/10 dilution). The signals were visualized using the following fluorochrome-coupled secondary antibodies: Alexa fluor 568 goat anti-mouse IgG1a (Invitrogen, cat\# A-21124), Alexa fluor 488 goat anti-rabbit IgG (Invitrogen, cat\# A-11008), and Alexa fluor 488 goat anti-rat IgG (Invitrogen, cat\# A-11006). Nuclei were counterstained with DAPI.

Stained cells or tissues were photographed using BIOREVO (Keyence) and quantified using Fiji (https://imagej.net/Fiji). The numbers of Uhrf1+, Pax7+, Ki67+, Myogenin+, and EdU+ cells in vitro and the area of Myh3+ fibers in vivo were determined using the Fiji Analyse Particles function. For total muscle CSA quantification, the whole area of transverse TA muscle sections was measured by laminin staining. The numbers of Uhrf1+, Pax7+, and EdU+ cells in vivo were

21 determined by manual counting using the Adobe Photoshop count tool.

\section{Isolation of satellite cells}

24 Isolation of satellite cells from hindlimb muscles was performed as described previously (Liu et al., 2015) with minor modifications. Briefly, muscles were chopped in cold PBS and digested using 800 
$1 \mathrm{U} / \mathrm{ml}$ collagenase type 2 (Worthington, cat\# LS004177) in F10 medium (Gibco, cat\# 11550-043)

2 supplemented with horse serum (Gibco, cat\# 26050-088) and antibiotic-antimycotic (Gibco, cat\# 3 15240-062) for $60 \mathrm{~min}$ followed by another digestion using $1000 \mathrm{U} / \mathrm{ml}$ collagenase type 2 and 11

$4 \mathrm{U} / \mathrm{ml}$ dispase (Gibco, cat\# 17105-041) for $30 \mathrm{~min}$. The supernatants were filtered through a $40 \mu \mathrm{m}$

5 cell strainer (Falcon, cat\# 352340) and centrifuged to yield a cell suspension. The following

6 antibodies were used: CD31-FITC (BioLegend, cat\# 102406; RRID:AB_312901; 1/100 dilution),

7 CD45-FITC (BioLegend, cat\# 103108, RRID:AB_312973; 1/100 dilution), Sca1-APC (BioLegend, cat\# 122512, RRID:AB_756197; 1/100 dilution), and Vcam1-PE (Invitrogen, cat\# 12-1061-82, RRID:AB_2572573; 1/100 dilution). Cells were isolated using the FACS Aria II (Becton, Dickinson and Company) and analyzed by FlowJo (https://www.flowjo.com).

\section{Cell culture, siRNA transfection, and DAPT treatment}

The $\mathrm{C} 2 \mathrm{C} 12$ cell line was culture in DMEM (Gibco, cat\# 10569-010) supplemented with 10\% FBS (Gibco, cat\# 10099-141) and antibiotic-antimycotic. The medium was replaced with differentiation medium (DMEM supplemented with $2 \%$ horse serum) to induce myotube formation. Isolated satellite cells were resuspended in DMEM/F12 (Gibco, cat\# 10565-018) supplemented with 20\% FBS, 2\% Ultroser G (Pall, cat\# 15950-017), and antibiotic-antimycotic. Cells at 5000 or $10000 / \mathrm{cm}^{2}$ were cultured on an 8 -well chamber slide (Thermo Scientific, cat\# 177445) or in a 6-well plate coated with $1 \mathrm{mg} / \mathrm{ml}$ Matrigel (Corning, cat\# 354230). Cells were transfected with non-targeting siRNA (Dharmacon, cat\# D-001810-10-05) or siRNA targeting

21 Cdkn1a (Dharmacon, cat\# L-058636-00-0005) using Lipofectamine RNAiMAX (Thermo cat\# 565784) in DMSO was used to inhibit Notch signaling. 


\section{EdU labelling and detection}

For in vivo labelling, EdU (5-ethynyl-2'-deoxyuridine, Invitrogen, cat\# A10044) was dissolved in

PBS at $0.5 \mu \mathrm{g} / \mu 1$ and injected intraperitoneally into mice at $5 \mu \mathrm{g} / \mathrm{g}$ body weight $24 \mathrm{~h}$ before harvesting. For in vitro labelling, the cells were incubated with EdU $(10 \mu \mathrm{M})$ for $3 \mathrm{~h}$. EdU was detected using Click-iT EdU Imaging Kits (Invitrogen, cat\# C10340) with Alexa Flour 647.

\section{RNA isolation and quantitative real-time PCR}

Total RNA was extracted from satellite cells using the RNeasy Plus Micro Kit (Qiagen, cat\# 74034)

9 following the manufacturer's protocol. cDNA was synthesized from total RNA using PrimeScript (Takara, cat\# RR036A). qPCR was performed in duplicate samples using TB Green Premix Ex Taq

II (Takara, cat\# RR820S) and Thermal Cycler Dice (Takara, cat\# TP850). The primer sequences are listed in Table 2.

\section{Isolation, culture, and immunostaining of single myofibers}

Single myofibers were isolated from extensor digitorum longus muscles as described previously

16 (Rosenblatt et al., 1995). The isolated fibers were fixed in 4\% PFA or cultured in DMEM supplemented with 30\% FBS, 1\% chicken embryo extract (US Biological, cat\# C3999), and 10 ng/ml bFGF (Wako, cat\# 062-06661). Immunostaining was performed following a previous protocol (Shinin et al., 2009).

\section{RNA-Seq and data analysis}

The integrity of isolated RNA was verified using the Agilent 2100 Bioanalyzer with RNA 6000 
1 E7335S) according to the manufacturer's instructions. The constructed libraries were verified using

2 the Agilent 2100 Bioanalyzer with DNA 1000 kit (Agilent, cat\# 5067-1504) and quantified using

3 KAPA Library Quantification Kits (KAPA Bio, cat\# KK4824) and the 7500 Real-Time PCR

4 System (ABI, cat\# 7500-01). The libraries were sequenced as 150 bp paired-end reads using the

5 NovaSeq 6000 Sequencing System (Illumina). The expression analysis was performed using

6 RaNA-Seq (Prieto and Barrios, 2019). Expression level was quantified by the Salmon algorithm

7 (Patro et al., 2017) using the paired-end, default parameters. Differential expression analysis was

8 performed by DESeq2 (Love et al., 2014) using the following parameters: test = Wald; fitType =

9 local. GO enrichment analysis was performed using Metascape (Zhou et al., 2019).

For transposable element (TE) analysis, raw reads were trimmed using TrimGalore (v0.6.6;

11 http://www.bioinformatics.babraham.ac.uk/projects/trim galore/) and mapped to the reference genome (GRCm38, PRI) with annotation (PRI, https://www.gencodegenes.org/mouse/) using STAR (v2.7.6a) (Dobin et al., 2013). The option '--runMode alignReads --runThreadN 4 outMultimapperOrder Random --outSAMtype BAM SortedByCoordinate --outSAMmultNmax 1 -outFilterMultimapNmax 1000 --outFilterMismatchNmax 3 --winAnchorMultimapNmax 1000 -alignIntronMax 1 --alignMatesGapMax 350 --alignEndsType EndToEnd' was used, allowing a higher number of multi-mapped reads (Teissandier et al., 2019). TE families were quantified using featureCounts (v2.0.1; -M -T 1 -s 0 -p) (Liao et al., 2014) and TE annotation differentially expressed TEs were extracted by DESeq2.

\section{MBD2-Seq and analysis of the sequencing data}

23 Genomic DNA was isolated and purified using the NucleoSpin Tissue Kit (MACHEREY-NAGEL, 24 cat\# 740952). Purified DNA was sheared using the Covaris S220 ultrasonicator with microTUBE 
1 the EpiXplore Methylated DNA Enrichment Kit (Clontech, cat\# PT5034-2). Libraries were generated using the DNA SMART ChIP-Seq Kit (Takara, cat\# 634865). The libraries were selected Sensitivity DNA kit (Agilent, cat\# 5067-4626) and quantified using KAPA Library Quantification Kits (KAPA Bio, cat\# KK4824) and the 7500 Real-Time PCR System (ABI, cat\# 7500-01). The libraries were sequenced using NovaSeq 6000 with 150 bp paired end reads. Raw reads were downsampled to 50 million reads for each condition to avoid the bias due to different sequencing depths.

9 The reads were trimmed using TrimGalore and were mapped using HISAT2 (Kim et al., 2015). Peaks were obtained by MACS2 (v2.2.6) (Zhang et al., 2008), using cells derived from TMXtreated mice as the 'control,' with the following options: --slocal 0 --llocal 0 -q 0.0001. ChIPpeakAnno (Zhu et al., 2010) was used to annotate and visualize the peaks. Methylated DNA signals were visualized using Integrative Genomics Viewer (IGV).

\section{Statistical analysis}

16 Data were analyzed using Prism 9 (GraphPad Software). Two-sample test for equality of proportions was performed by prop.test in R (https://www.r-project.org). Welch's t-test was used to compare variable between two groups. Welch's ANOVA with Dunnett's T3 multiple comparison test was used to compare variables among three groups.

\section{Data availability} number GSE169193. 


\section{Study approval}

All animals were maintained and used according to a protocol approved by the Animal Experiment Committee of Ehime University, Japan. differentiation ( $\mathrm{n}=3$ for each time point).

Supplementary Figure 2. Deletion of Uhrf1 in satellite cells in $\operatorname{Pax} 7^{C E /+} ; U h r f 1^{f l f l}$ mice disturbs proliferation and differentiation of satellite cells. (A) Experimental design for TMX followed by FACS and immunostaining after 3 days in culture. (B) Representative FACS profiles of satellite cells from oil- or TMX-treated $\operatorname{Pax} 7^{C E /+} ; U h r f 1^{f l f l}$ mice. (C) RT-qPCR analysis of Uhrfl in satellite cells from oil- or TMX-treated $P a x 7^{C E /+} ; U h r f 1^{f l f l}$ mice ( $\mathrm{n}=3$ mice/condition). (D) Quantification of Uhrf1+ cells by IF ( $n=362$ cells for oil-treated mice, $n=304$ cells for TMX-treated mice). Data are expressed as the mean with $95 \%$ CI. (E) Immunostaining of Uhrf1 in satellite cells from oil- or (G) The average of total area in TA muscle cross-sections at $5 \mathrm{dpi}(\mathrm{n}=3 \mathrm{mice} /$ condition $) .(\mathrm{H})$ Immunostaining of laminin in TA at 28 dpi. (I) Experimental design for TMX followed by isolating single fibers. (J) Quantification of Pax7+ and Myod+ cells by immunostaining during myogenic differentiation in single fibers $(n=150-600$ cells for each group, data represent the mean $\pm S D)$. $(K)$ Experimental design for TMX followed by FACS and IF after 3, 4, and 7 days in culture. (L) EdU detection in satellite cells from oil- or TMX-treated $\operatorname{Pax}^{\mathrm{CE} /{ }^{+}} ; U h r f f^{f l / f l}$ mice after 3 days in culture. (M) Quantification of EdU+ cells after 3 days in culture. (N) Immunostaining of Myogenin after 4 days in culture. (O) Quantification of Myogenin+ cells after 4 days in culture. (P) Immunostaining 
1 of Myh1 after 7 days in culture. (Q) Fusion index after 7 days in culture. For graphs in D, M, O, and

2 Q, data were pooled from three independent experiments and data are expressed as the mean with 95\% CI. Welch's t-test (C and J), two-sample test for equality of proportions with continuity

4 correction (D, M, O, and Q), ${ }^{*} p<0.05,{ }^{*} p<0.01, * * * p<0.001, * * * * p<0.0001$. Scale bars $=50$ $5 \mu \mathrm{m}$ in $(\mathrm{E}), 500 \mu \mathrm{m}$ in $(\mathrm{H})$, and $100 \mu \mathrm{m}$ in $(\mathrm{L}),(\mathrm{N})$, and $(\mathrm{P})$.

Supplementary Figure 3. Loss of Uhrf1 in satellite cells induces a distinct gene expression profile, including transposable elements (TEs), and alters the DNA methylation pattern. (A)

Heatmap of the expression similarities among samples. (B) MA of the differential expression results. Genes with a significant change in expression are highlighted as red dots. (C) Venn

11 Diagram showing the genes commonly up-regulated by deletion of Uhrf1 among satellite cells, chondrocytes (GSE92641), and hematopoietic stem cells (GSE85450). (D) MA of differential expression of TEs. (E) Up-regulated TEs in mutant satellite cells. (F) GC content per read of methylated and non-methylated DNA extracted from control satellite cells. (G) Distinct methylation patterns observed in H19 and Kcnq1ot1 genes in methylated and non-methylated DNA from control satellite cells. (H) Boxplot showing the expression distribution (normalized as TPMs) of DNA methylation-related genes in each group.

\section{Supplementary Figure 4. Uhrf1 expression in satellite cells is not altered by knockdown of}

Cdkn1a or DAPT treatment. (A) RT-qPCR analysis of Cdknla in satellite cells from control and mutant mice treated with $C d k n l a$ siRNA ( $=6$ mice/condition). (B) RT-qPCR analysis of HeyL in satellite cells from control and mutant mice treated with DAPT $(n=4 /$ condition $)$. (C) 
bioRxiv preprint doi: https://doi.org/10.1101/2021.04.08.439096; this version posted April 10, 2021. The copyright holder for this preprint (which was not certified by peer review) is the author/funder. All rights reserved. No reuse allowed without permission.

1 Immunostaining of Uhrf1 in satellite cells treated with DAPT. (G) Quantification of Uhrf1+ DAPT-

2 treated satellite cells ( $\mathrm{n}=3$ mice/condition). One-way ANOVA with Šídák's multiple comparison

3 test was performed, ${ }^{*} p<0.05, * * p<0.01, * * * * p<0.0001$. Scale bars $=50 \mu \mathrm{m}$. 


\section{References}

Blanchart, A., Navis, A.C., Assaife - Lopes, N., Usoskin, D., Aranda, S., Sontheimer, J., and Ernfors, P. (2018). UHRF1 licensed self-renewal of active adult neural stem cells. Stem Cells 36, 1736-1751.

Bostick, M., Kim, J.K., Estève, P.-O., Clark, A., Pradhan, S., and Jacobsen, S.E. (2007). UHRF1 plays a role in maintaining DNA methylation in mammalian cells. Science 317, 1760-1764. carrió, E., Díez - Villanueva, A., Lois, S., Mallona, I., Cases, I., Forn, M., Peinado, M.A., and Suelves, M. (2015). Deconstruction of DNA methylation patterns during myogenesis reveals specific epigenetic events in the establishment of the skeletal muscle lineage. Stem Cells 33, 2025-2036.

Dobin, A., Davis, C.A., Schlesinger, F., Drenkow, J., Zaleski, C., Jha, S., Batut, P., Chaisson, M., and Gingeras, T.R. (2013). STAR: ultrafast universal RNA-seq aligner. Bioinformatics 29, 15-21. Fukada, S., Uezumi, A., Ikemoto, M., Masuda, S., Segawa, M., Tanimura, N., Yamamoto, H., Miyagoe - Suzuki, Y., and Takeda, S. (2007). Molecular signature of quiescent satellite cells in adult skeletal muscle. Stem Cells 25, 2448-2459.

Fukada, S., Yamaguchi, M., Kokubo, H., Ogawa, R., Uezumi, A., Yoneda, T., Matev, M.M., Motohashi, N., Ito, T., Zolkiewska, A., et al. (2011). Hesr1 and Hesr3 are essential to generate undifferentiated quiescent satellite cells and to maintain satellite cell numbers. Development 138, 4609-4619.

Gerli, M.F.M., Moyle, L.A., Benedetti, S., Ferrari, G., Ucuncu, E., Ragazzi, M., Constantinou, C., Louca, I., Sakai, H., Ala, P., et al. (2019). Combined Notch and PDGF signaling enhances migration and expression of stem cell markers while inducing perivascular cell features in muscle satellite cells. Stem Cell Rep 12, 461-473.

Iio, H., Kikugawa, T., Sawada, Y., Sakai, H., Yoshida, S., Yanagihara, Y., Ikedo, A., Saeki, N., Fukada, S., Saika, T., et al. (2021). DNA maintenance methylation enzyme Dnmt1 in satellite cells is essential for muscle regeneration. Biochem Bioph Res Co 534, 79-85. Kim, D., Langmead, B., and Salzberg, S.L. (2015). HISAT: a fast spliced aligner with low memory requirements. Nat Methods $12,357-360$.

Lepper, C., Conway, S.J., and Fan, C.-M. (2009). Adult satellite cells and embryonic muscle progenitors have distinct genetic requirements. Nature 460, 627-631.

Lepper, C., Partridge, T.A., and Fan, C.-M. (2011). An absolute requirement for Pax7-positive satellite cells in acute injury-induced skeletal muscle regeneration. Dev Camb Engl 138, 36393646.

Liao, Y., Smyth, G.K., and Shi, W. (2014). featureCounts: an efficient general purpose program for assigning sequence reads to genomic features. Bioinformatics 30, 923-930.

Liu, L., Cheung, T.H., Charville, G.W., and Rando, T.A. (2015). Isolation of skeletal muscle stem cells by fluorescence-activated cell sorting. Nat Protoc 10,1612-1624.

Love, M.I., Huber, W., and Anders, S. (2014). Moderated estimation of fold change and dispersion for RNA-seq data with DESeq2. Genome Biol 15, 550.

Mademtzoglou, D., Asakura, Y., Borok, M.J., Alonso-Martin, S., Mourikis, P., Kodaka, Y., Mohan, A., Asakura, A., and Relaix, F. (2018). Cellular localization of the cell cycle inhibitor Cdkn1c controls growth arrest of adult skeletal muscle stem cells. Elife 7, e33337.

McCarthy, J.J., Mula, J., Miyazaki, M., Erfani, R., Garrison, K., Farooqui, A.B., Srikuea, R., Lawson, B.A., Grimes, B., Keller, C., et al. (2011). Effective fiber hypertrophy in satellite cell-depleted skeletal muscle. Development 138, 3657-3666.

Meilinger, D., Fellinger, K., Bultmann, S., Rothbauer, U., Bonapace, I.M., Klinkert, W.E.F., Spada, F., and Leonhardt, H. (2009). Np95 interacts with de novo DNA methyltransferases, Dnmt3a 
and Dnmt3b, and mediates epigenetic silencing of the viral CMV promoter in embryonic stem cells. Embo Rep 10, 1259-1264.

Miyata, K., Miyata, T., Nakabayashi, K., Okamura, K., Naito, M., Kawai, T., Takada, S., Kato, K., Miyamoto, S., Hata, K., et al. (2015). DNA methylation analysis of human myoblasts during in vitro myogenic differentiation: de novo methylation of promoters of muscle-related genes and its involvement in transcriptional down-regulation. Hum Mol Genet 24, 410-423.

Morgan, H.D., Sutherland, H.G.E., Martin, D.I.K., and Whitelaw, E. (1999). Epigenetic inheritance at the agouti locus in the mouse. Nat Genet 23, 314-318.

Mudbhary, R., Hoshida, Y., Chernyavskaya, Y., Jacob, V., Villanueva, A., Fiel, M.I., Chen, X., Kojima, K., Thung, S., Bronson, R.T., et al. (2014). UHRF1 overexpression drives DNA hypomethylation and hepatocellular carcinoma. Cancer Cell 25, 196-209.

Murphy, M.M., Lawson, J.A., Mathew, S.J., Hutcheson, D.A., and Kardon, G. (2011). Satellite cells, connective tissue fibroblasts and their interactions are crucial for muscle regeneration. Development 138, 3625-3637.

Naito, M., Mori, M., Inagawa, M., Miyata, K., Hashimoto, N., Tanaka, S., and Asahara, H. (2016). Dnmt3a regulates proliferation of muscle satellite cells via p57Kip2. Plos Genet 12, e1006167. Obata, Y., Furusawa, Y., Endo, T.A., Sharif, J., Takahashi, D., Atarashi, K., Nakayama, M., Onawa, S., Fujimura, Y., Takahashi, M., et al. (2014). The epigenetic regulator Uhrf1 facilitates the proliferation and maturation of colonic regulatory T cells. Nat Immunol 15, 571-579. Patro, R., Duggal, G., Love, M.I., Irizarry, R.A., and Kingsford, C. (2017). Salmon provides fast and bias-aware quantification of transcript expression. Nat Methods 14, 417-419.

Prieto, C., and Barrios, D. (2019). RaNA-Seq: Interactive RNA-Seq analysis from FASTQ files to functional analysis. Bioinformatics.

Ramesh, V., Bayam, E., Cernilogar, F.M., Bonapace, I.M., Schulze, M., Riemenschneider, M.J., Schotta, G., and Götz, M. (2016). Loss of Uhrf1 in neural stem cells leads to activation of retroviral elements and delayed neurodegeneration. Gene Dev 30, 2199-2212.

Rosenblatt, J.D., Lunt, A.I., Parry, D.J., and Partridge, T.A. (1995). Culturing satellite cells from living single muscle fiber explants. Vitro Cell Dev Biology - Animal 31, 773-779.

Sakai, H., Sato, T., Kanagawa, M., Fukada, S., and Imai, Y. (2020). Androgen receptor in satellite cells is not essential for muscle regenerations. Exp Results 1.

Sambasivan, R., Yao, R., Kissenpfennig, A., Wittenberghe, L.V., Paldi, A., Gayraud-Morel, B., Guenou, H., Malissen, B., Tajbakhsh, S., and Galy, A. (2011). Pax7-expressing satellite cells are indispensable for adult skeletal muscle regeneration. Development 138, 3647-3656.

Seale, P., Sabourin, L.A., Girgis-Gabardo, A., Mansouri, A., Gruss, P., and Rudnicki, M.A. (2000). Pax7 is required for the specification of myogenic satellite cells. Cell 102, 777-786.

Sharif, J., Muto, M., Takebayashi, S., Suetake, I., Iwamatsu, A., Endo, T.A., Shinga, J., MizutaniKoseki, Y., Toyoda, T., Okamura, K., et al. (2007). The SRA protein Np95 mediates epigenetic inheritance by recruiting Dnmt1 to methylated DNA. Nature 450, 908-912.

Shinin, V., Gayraud-Morel, B., and Tajbakhsh, S. (2009). Stem cells in regenerative medicine. Methods Mol Biology Clifton N J 482, 295-317.

Skarnes, W.C., Rosen, B., West, A.P., Koutsourakis, M., Bushell, W., Iyer, V., Mujica, A.O., Thomas, M., Harrow, J., Cox, T., et al. (2011). A conditional knockout resource for the genomewide study of mouse gene function. Nature 474, 337-342.

Teissandier, A., Servant, N., Barillot, E., and Bourc'his, D. (2019). Tools and best practices for retrotransposon analysis using high-throughput sequencing data. Mobile Dna-Uk 10, 52. Tsumagari, K., Baribault, C., Terragni, J., Varley, K.E., Gertz, J., Pradhan, S., Badoo, M., Crain, C.M., Song, L., Crawford, G.E., et al. (2013). Early de novo DNA methylation and prolonged demethylation in the muscle lineage. Epigenetics 8, 317-332. 
1 Wen, Y., Bi, P., Liu, W., Asakura, A., Keller, C., and Kuang, S. (2012). Constitutive Notch activation upregulates Pax7 and promotes the self-renewal of skeletal muscle satellite cells. Mol Cell Biol 32, 2300-2311.

4 Xiang, H., Yuan, L., Gao, X., Alexander, P.B., Lopez, O., Lau, C., Ding, Y., Chong, M., Sun, T., Chen, $\mathrm{R}$., et al. (2017). UHRF1 is required for basal stem cell proliferation in response to airway injury. Cell Discov 3, 17019.

7 Yamashita, M., Inoue, K., Saeki, N., Ideta-Otsuka, M., Yanagihara, Y., Sawada, Y., Sakakibara, I., 8 Lee, J., Ichikawa, K., Kamei, Y., et al. (2017). Uhrf1 is indispensable for normal limb growth by 9 regulating chondrocyte differentiation through specific gene expression. Development 145, 10 dev.157412. Zhang, Y., Liu, T., Meyer, C.A., Eeckhoute, J., Johnson, D.S., Bernstein, B.E., Nusbaum, C., Myers, R.M., Brown, M., Li, W., et al. (2008). Model-based analysis of ChIP-Seq (MACS). Genome Biol 9, R137.

Zhao, J., Chen, X., Song, G., Zhang, J., Liu, H., and Liu, X. (2017). Uhrf1 controls the self-renewal versus differentiation of hematopoietic stem cells by epigenetically regulating the celldivision modes. Proc National Acad Sci 114, E142-E151. Zhou, Y., Zhou, B., Pache, L., Chang, M., Khodabakhshi, A.H., Tanaseichuk, O., Benner, C., and Chanda, S.K. (2019). Metascape provides a biologist-oriented resource for the analysis of systems-level datasets. Nat Commun 10, 1523. 


\section{Pax $7^{\mathrm{CE} / \mathrm{t}} ;$ Uhrf $^{f / f}$}

Oil

TMX FACS

RNA-Seq

$\downarrow$ MBD2-Seq

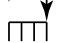

03 days

B

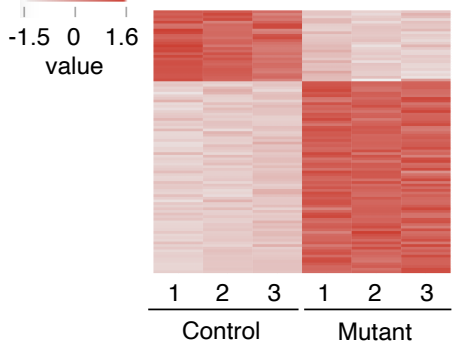

$\mathbf{F}$
- $\log 10(p-$ value $)$
0

vasculature development

artery morphogenesis

DNA damage response, signal transduction by p53 class mediator resulting in cell cycle arrest

regulation of protein catabolic process

regulation of protein transport

regulation of cellular response to stress

\section{Up-regulated in Mutant}

D

\begin{tabular}{|l|}
\multicolumn{1}{l}{$-\log 10$ (q-value) } \\
\hline vasculature development \\
\hline positive regulation of hydrolase activity \\
\hline regulation of cell adhesion \\
\hline actin filament-based process \\
\hline positive regulation of proteolysis \\
\hline apoptotic signaling pathway \\
\hline
\end{tabular}

\section{Down-regulated in Mutant}

\begin{tabular}{l|}
\multicolumn{2}{l}{$-\log 10(\mathrm{q}-\mathrm{value})$} \\
\hline ribonucleoprotein complex biogenesis \\
\hline mRNA metabolic process \\
\hline mitotic cell cycle process \\
\hline chromosome segregation \\
\hline DNA replication \\
\hline DNA repair \\
\hline
\end{tabular}

G

80

E
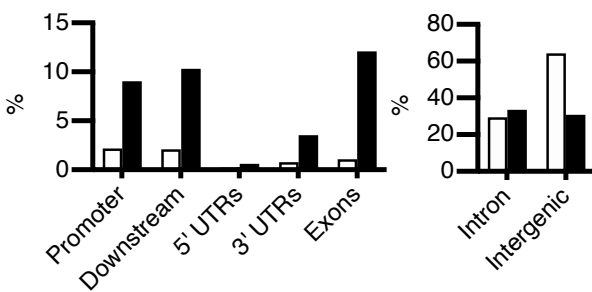
Up-regulated genes

Genes with Uhrf1-mediated DNA methylated peaks

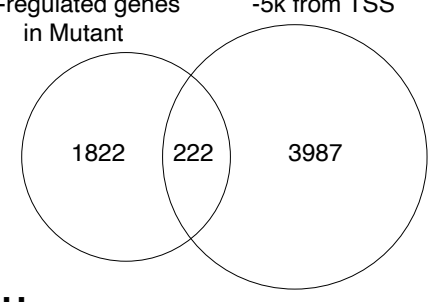

H

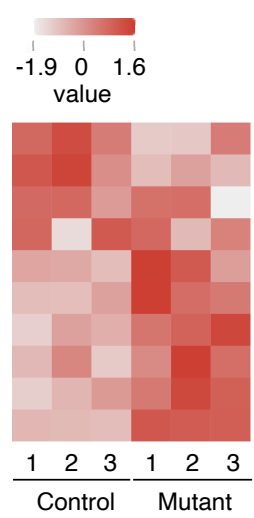

Myog*

Myod1

Myf5*

Notch1

Pax7

Notch2

Hes1

Heyl $^{*}$

Hey1

Notch3
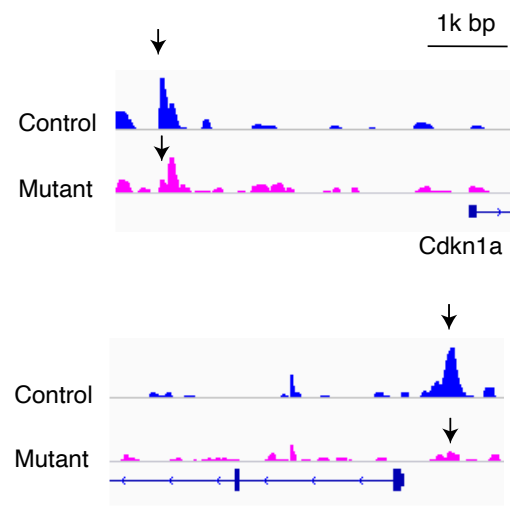

Notch3 


\section{A}

$\operatorname{Pax} 7^{\mathrm{CE} /+} ;$ Uhrf $1^{f|/| t \mid}$
Oil

TMX thrtiाtiाt
RNA

DAPT

EdU (3h)

\section{B}

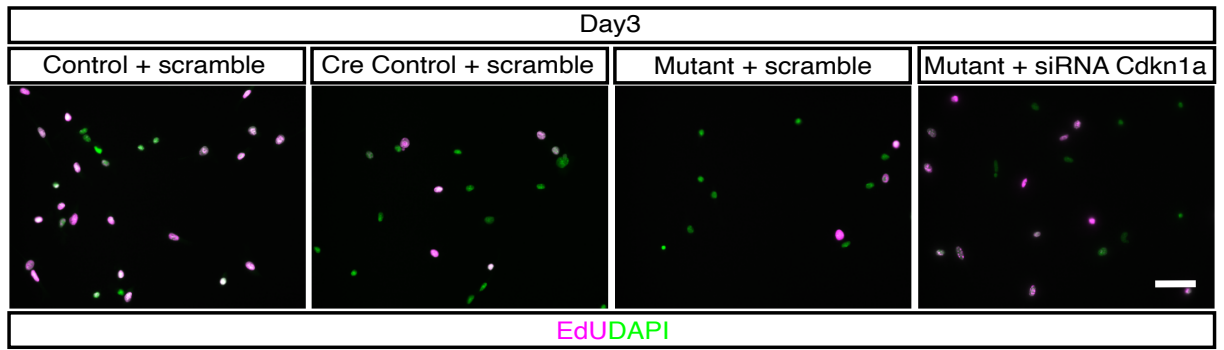

D

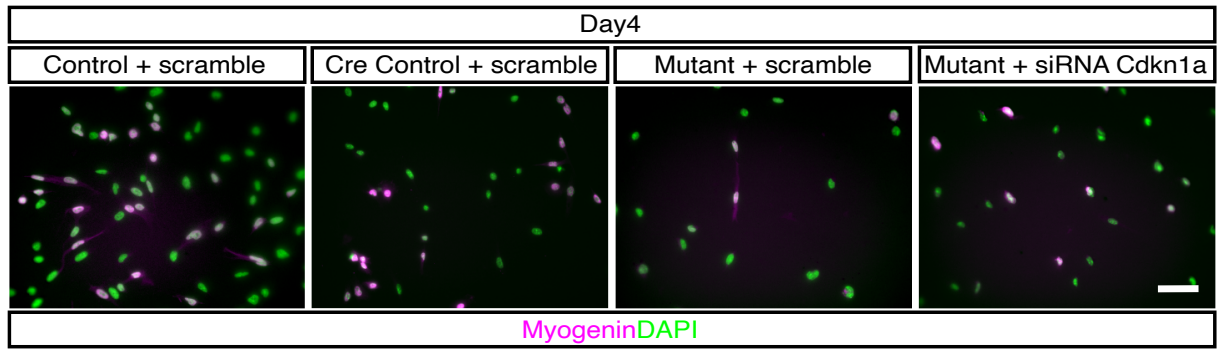

$\mathbf{F}$

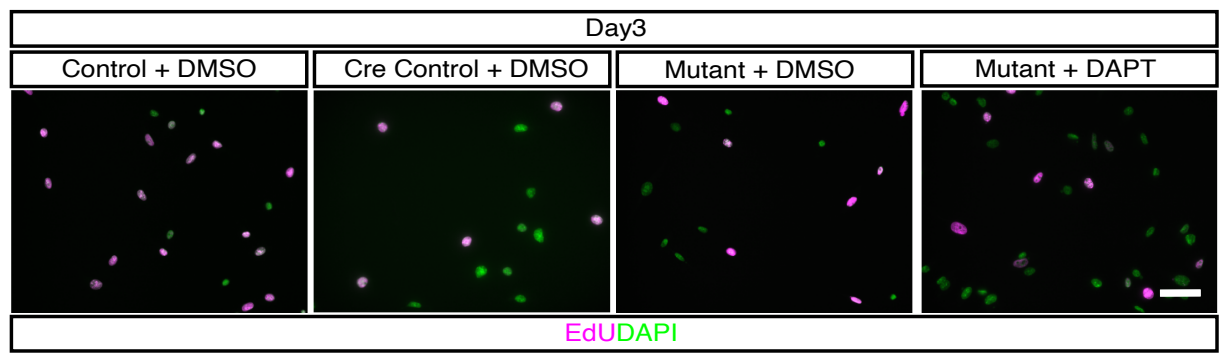

H

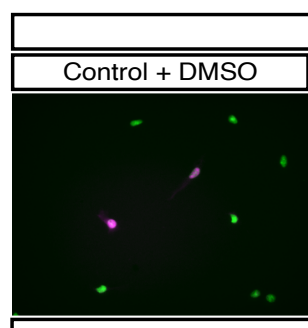

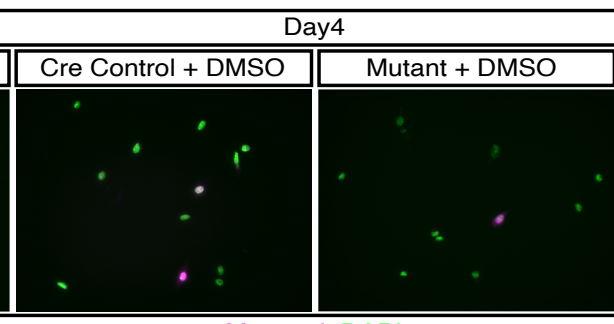

MyogeninDAPI

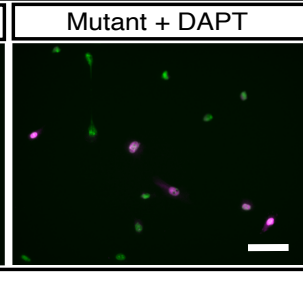

C

O Control + scramble

$\triangle \quad$ Cre Control + scramble

$\square \quad$ Mutant + scramble

- Mutant + siRNA Cdkn1a

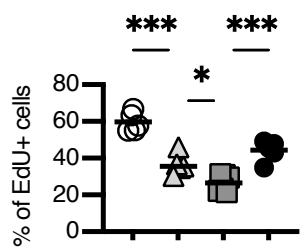

E

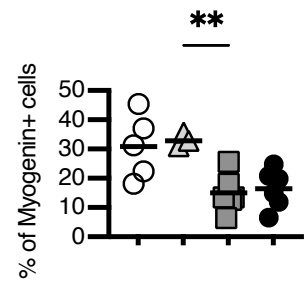

G

O Control + DMSO

$\triangle \quad$ Cre Control + DMSO

$\square \quad$ Mutant + DMSO

- Mutant + DAPT
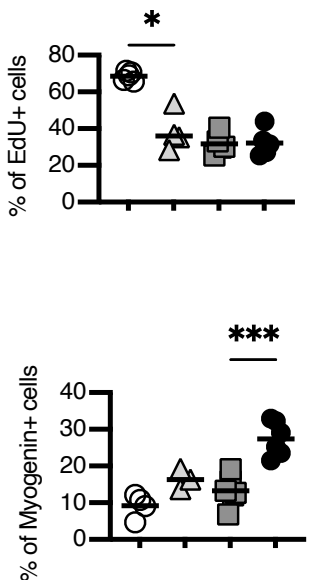

\section{I}


A
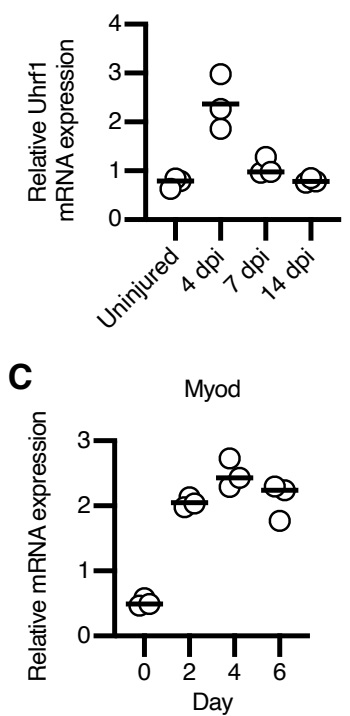

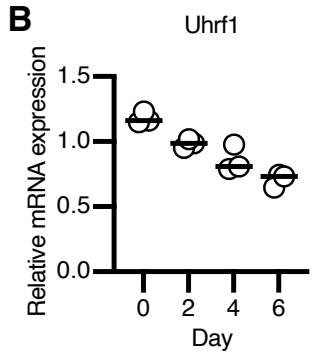

D Myogenin

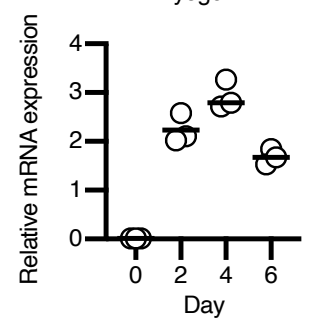

Figure S1 

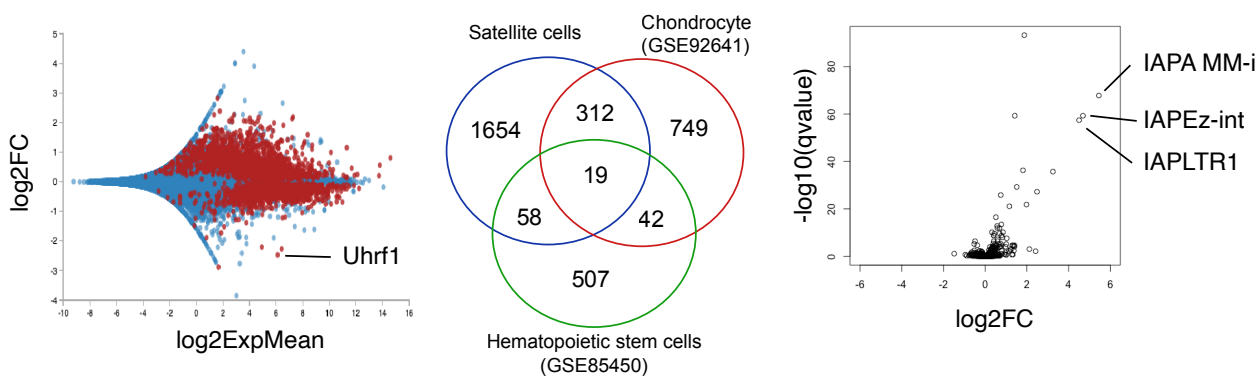

E

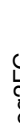
से

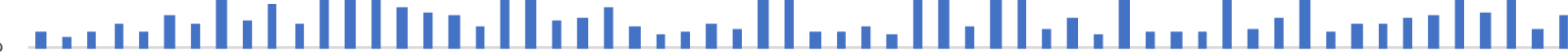

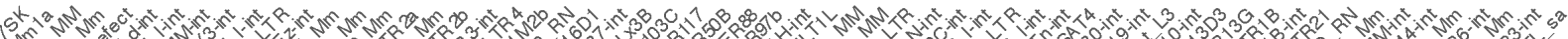

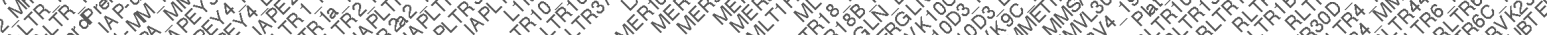

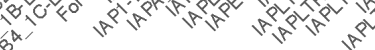

$\mathbf{F}$

GC count per read

Theorectical Distribution

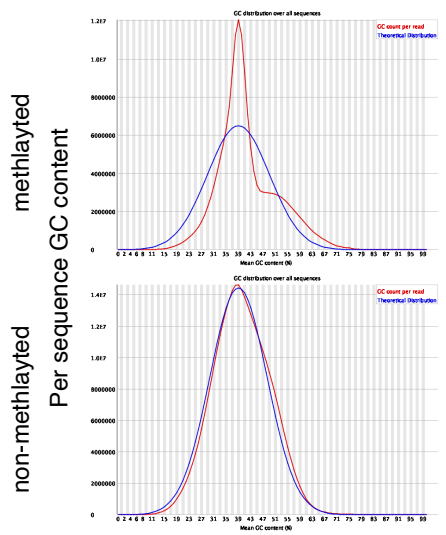

Mean GC content (\%)
G

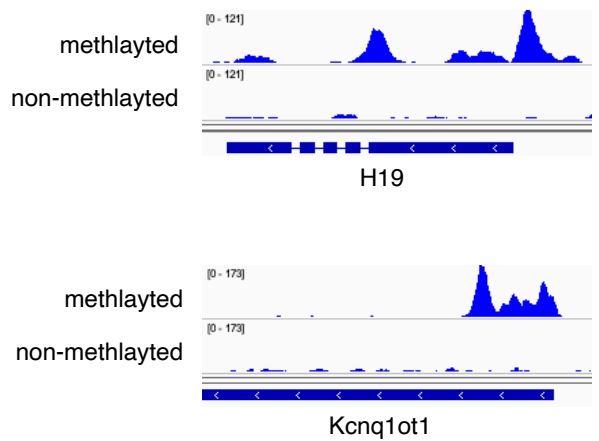

$\mathbf{H}$

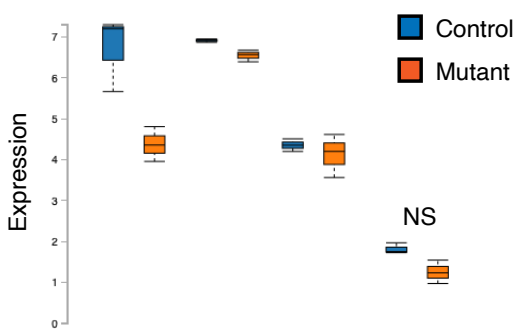

Uhrf1 Dnmt1 Dnmt3a Dnmt3b 
